\title{
$\mathrm{RGB}$ 영상에서 딥러닝 기반 동공 중심점을 이용한 홍채 검출 \\ 이태균*, 유장희 $* * \dagger$
}

\section{Iris Localization using the Pupil Center Point based on Deep Learning in RGB Images}

\author{
Tae-Gyun Lee*, Jang-Hee Yoo**†
}

요 약

본 논문에서는 RGB 영상에서 홍채 검출 방법에 관하여 기술하였다. 기존의 홍채 검출 방법은 대부분 적 외선 영상을 대상으로 하고 있어, 다양한 응용을 위해서는 RGB 영상의 홍채 검출 기술이 요구된다. 제안된 홍채 검출 방법은 1) 입력 영상에서 원형 허프 변환을 사용한 홍채 후보 영역 검출, il) 딥러닝 기반의 동공 중심점 검출, iii) 동공 중심점을 이용한 홍채 영역 선택, $i v)$ 선택된 홍채 영역 보정 과정으로 구성된다. 홍채 후보 영역은 허프 공간을 생성한 후 중심점 후보의 교차 개수가 많은 순으로 검출하며, 후보 영역 중 홍채는 검출된 동공의 중심점을 기준으로 선택한다. 그리고, 홍채의 모양이 왜곡되어 오차가 발생하는 것을 보완하기 위해 검출된 홍채 중심을 기준으로 새로운 경계점을 찾아 보정하는 방법을 사용하였다. 또한, 실험을 통하여 제안된 방법이 기존 원형 허프 변환 방법 대비 약 $27.4 \%$ 향상된 정확도를 갖는 것을 확인하였다.

\begin{abstract}
In this paper, we describe the iris localization method in RGB images. Most of the iris localization methods are developed for infrared images, thus an iris localization method in RGB images is required for various applications. The proposed method consists of four stages: 1) detection of the candidate irises using circular Hough transform (CHT) from an input image, ii) detection of a pupil center based on deep learning, iii) determine the iris using the pupil center, and iv) correction of the iris region. The candidate irises are detected in the order of the number of intersections of the center point candidates after generating the Hough space, and the iris in the candidates is determined based on the detected pupil center. Also, the error due to distortion of the iris shape is corrected by finding a new boundary point based on the detected iris center. In experiments, the proposed method has an improved accuracy about $27.4 \%$ compared to the CHT method.
\end{abstract}

한글키워드 : 홍채 검출, 동공 검출, 홍채인식, 딥러닝, 허프 변환

keywords : iris localization, pupil detection, iris recognition, deep learning, hough transform

* 과학기술연합대학원대학교 ICT전공

** 한국전자통신연구원 인공지능연구소

† 교신저자: 유장희(email: jhy@etri.re.kr) 접수일자: 2020.11.25. 심사완료: 2020.12.08. 게재확정: 2020.12.21.

\section{1. 서 론}

홍채인식은 적외선 안구 영상을 획득하여, 동 공(pupil)과 공막(sclera) 사이의 도넛 모양 근육 
조직인 홍채(iris)의 패턴을 이용한 개인 식별기 술이다. 안구 영상을 획득하기 위해서는 적외선 카메라뿐 아니라 적외선 조명도 함께 사용하여야 한다. 이는 일반적으로 안구의 멜라닌(melanin) 은 가시광선을 흡수하는 데 비하여, 적외선 조명 의 대부분을 반사함으로써 더욱 선명한 홍채 영 상을 획득할 수 있는데 기인한다[1]. 사람의 홍채 는 생후 18 개월이면 완성되어, 평생 변하지 않는 특성이 있다. 쌍둥이도 서로 다른 홍채 패턴을 보이며, 양안 모두 서로 다른 패턴을 보이는 등 가장 정확도가 높은 생체인식 기술이다[1][2]. 최 근 들어, 임베디드 플랫폼 기반의 Stand-alone 제품뿐만 아니라 각종 모바일기기 등에서도 홍채 인식 기술을 적용하려는 시도와 함께, 적외선 카 메라와 조명을 추가하지 않고도 기존의 $\mathrm{RGB}$ 카 메라 기반의 홍채인식 기술에 관한 연구가 꾸준 히 진행되고 있다[1][3][4].

홍채인식 과정에서 가장 중요한 기술 중의 하 나는 홍채를 정확하게 검출하는 것이다. 홍채 검 출에서 가장 널리 사용되는 방법은 허프 변환 (Hough Transform) 기반의 방법[5], Daugman의 미적분 연산 기반의 방법[6], $\mathrm{Ma}$ 의 동공 검출 기 반의 방법[7] 등이 있다. 이와 같은 방법들은 대 부분 적외선 영상을 기반으로 하고 있어, $\mathrm{RGB}$ 영상에 그대로 적용할 경우는 홍채가 제대로 검 출되지 않는다. 이러한 문제를 해결하기 위해, Arsalan 등은 $\mathrm{RGB}$ 영상에서 원형 허프 변환 (CHT: Circular Hough Transform)을 적용하여 홍채 영역을 대략 검출하고, $\mathrm{CNN}$ 학습 기반으로 정확한 홍채 영역을 검출하는 방법을 제안하였다 [8]. 즉, $\mathrm{CNN}$ 의 연산량을 줄이기 위해, 허프 변환 으로 검출된 영역을 $\mathrm{RoI}$ (Region of Interest)로 설정하였고, $\mathrm{RoI}$ 를 $21 \times 21$ 크기로 분할한 영상을 $\mathrm{CNN}$ 에 입력으로 훈련한 모델을 사용하였다. 그 러나, $\mathrm{RGB}$ 영상에서의 홍채 검출은 여전히 해결 하기 어렵고 도전적인 문제이다.
본 연구에서는 이러한 배경을 토대로 앞서 언 급한 Arsalan 등에 의한 $\mathrm{CHT}$ 방법으로 검출된 다수의 홍채 후보 영역 중에서 최선의 홍채 영역 을 선택하기 위해 딥러닝 모델을 사용하였다. 즉, 시선(gaze) 검출 과정에서 동공(pupil) 중심점 검 출을 위해 사용된 딥러닝 모델[9]을 적용하여, $\mathrm{CHT}$ 에 의한 다수의 홍채 후보 영역 중에서 동 공 중심점이 있는 경우를 홍채 영역으로 판단하 는 방법을 제안하였다. 또한, 정확한 홍채의 경계 영역 분할을 위하여, $\mathrm{AIPF}$ (Angular Integral Projection Function)를 기반으로 검출된 홍채 영 역을 타원 형태로 근사하고, 이를 보정하는 방법 을 사용하였다[10]. 그리고, 제안된 방법의 성능 검증을 위하여, $\mathrm{CHT}$ 기반 홍채 검출 방법과 제 안된 방법의 홍채 검출 성능을 비교하는 실험을 수행하였다. 실험에서 제안된 방법이 기존 방법 과 비교해 정확도가 높음을 확인할 수 있었다.

\section{RGB 영상에서 홍채 검출}

본 논문에서 제안한 홍채 검출 과정은 그림 1 과 같다. 먼저 입력 영상에서 홍채 후보 영역을 검출하기 위해, 원형 허프 변환 $(\mathrm{CHT})$ 방법을 사 용하였다. 허프 변환을 위해서는 에지(edge) 영 상이 필요하므로 $\mathrm{RGB}$ 영상을 그레이(gray) 영상 으로 변환한 후 에지를 검출하였다. 이때 에지 영상의 홍채 영역 주변부에서 발생하는 노이즈를 감소시키기 위하여, 변환된 그레이 영상에 히스 토그램 평활화와 가우시안 블러링의 전처리 과정 을 수행하였다. 이와 같은 과정으로 검출된 에지 는 원형 허프 변환을 통해 홍채 후보 영역들을 검출하는 데 사용된다. 그리고, 여러 개의 홍채 후보 중 홍채를 선택하기 위해, 딥러닝 모델을 기반으로 검출된 동공 중심 정보를 사용하였다. 또한, 검출된 홍채의 부정확한 경계를 기준으로 
새로운 경계점을 찾고, 타원 형태로 보정하여 보 다 정확한 홍채를 검출할 수 있다. 다음의 각 절 에서는 본 논문에서 제안된 방법의 각 단계를 자 세히 설명하였다.

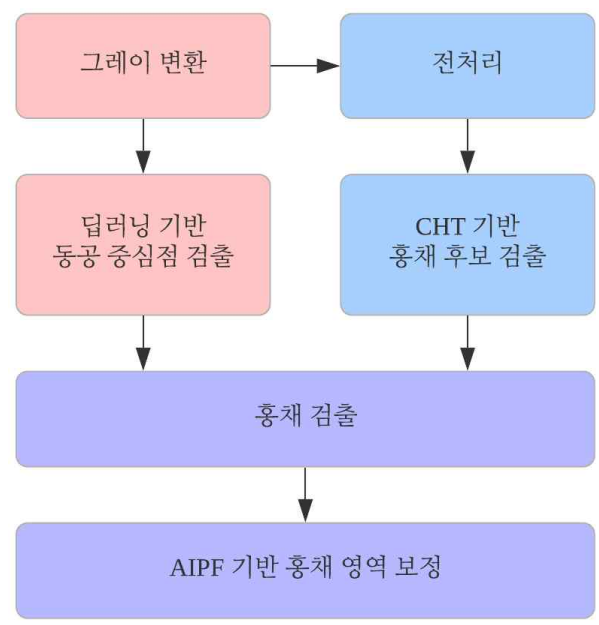

그림 1. 홍채 검출 과정

Fig. 1. Iris Localization Process

\section{1 $\mathrm{CHT}$ 기반의 홍채 후보 영역 검출}

$\mathrm{RGB}$ 영상으로부터 생성된 에지 영상은 원형 허 프 변환을 통해 홍채 후보를 검출하는 데 사용된 다. 일반적으로 하나의 원을 결정짓기 위해서는 원 의 중심점 $(a, b)$ 와 반지름 $r$ 이 필요하다. 원형 허프 변환 과정에서 반지름은 설정된 범위 내에서 하나 의 값으로 고정되며, 검출된 모든 에지에서 원 모 양의 중심점 후보들을 나타내어 홍채의 중심점을 결정하게 된다[12]. 그리고, 원 위의 모든 점에서 중심점 후보는 원의 중심점에서 교차하게 된다. 따 라서, 에지 영상의 각 픽셀에서 중심점 후보가 교 차하는 개수를 값으로 하는 허프 공간(Hough Space)을 생성하고, 값이 큰 것부터 차례로 선택 하여 홍채 후보 영역들을 검출할 수 있다. 그림 2 는 입력 영상에서 홍채 후보를 10 개씩 검출하여 표시한 예이다.

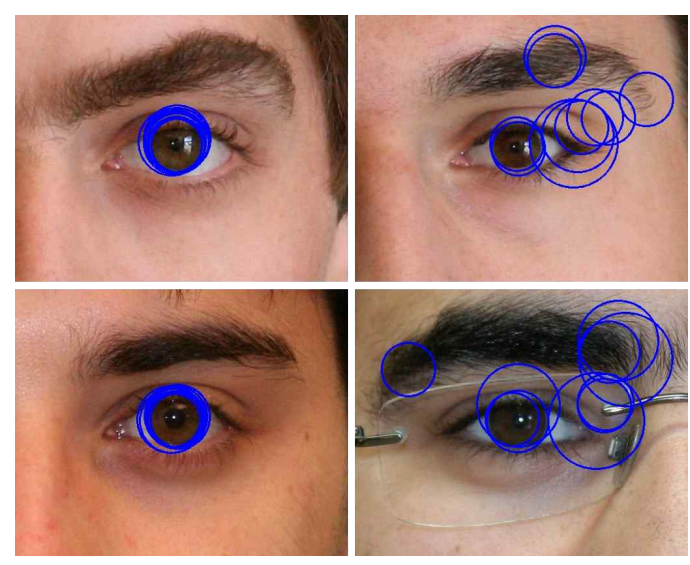

그림 2. 홍채 후보 검출 결과

Fig. 2. Results for Detection of the Iris Candidates

\section{2 동공 중심 기반의 홍채 영역 선택}

입력된 얼굴 영상에는 홍채와 유사한 특징을 갖는 부분들이 있다. 예를 들어, 눈 주변 영역의 눈썹, 코, 점 등에서 나타나는 유사 특징으로 에 지 영상으로부터 검출된 여러 개의 홍채 후보 중 에는 홍채 영역에서 완전히 벗어난 것이 있을 수 있다. 따라서 정확한 동공 중심점을 검출할 수 있다면, 이를 기준으로 홍채 후보의 중심 좌표 중에서 최소 거리에 해당하는 경우를 홍채로 선 택하는 방법을 사용할 수 있다.

본 논문에서는 동공 중심점 검출을 위하여, 그 림 3과 같은 딥러닝 기반의 DeepEye 모델을 사 용하였다[9]. 그림에서 Conv. 층(layer)과 Strided Conv. 층은 Residual과 함께 두 개의 합성 곱 층 으로 구성되며, Strided Conv. 층을 통해 영상의 크기를 축소한다. ASPP(Atrous Spatial Pyramid Pooling)[11] 층에서는 입력 영상에 대해 Atrous Convolution(Dilated Convolution)과 $1 \times 1$ Convolution, Max-Pooling을 동시에 수행한 뒤 모든 결과를 합친다. 그리고, Conv. $1 \times 1$ 을 통해 채널의 수를 감소시키고, 마지막으로 Bilinear $\mathrm{Up}^{-}$sampling을 통해 출력의 크기를 입력과 같게 
한다. 그림에서 $L$ 은 채널의 개수를 의미하며, Strided Conv. 층에서 2배로 증가한다.

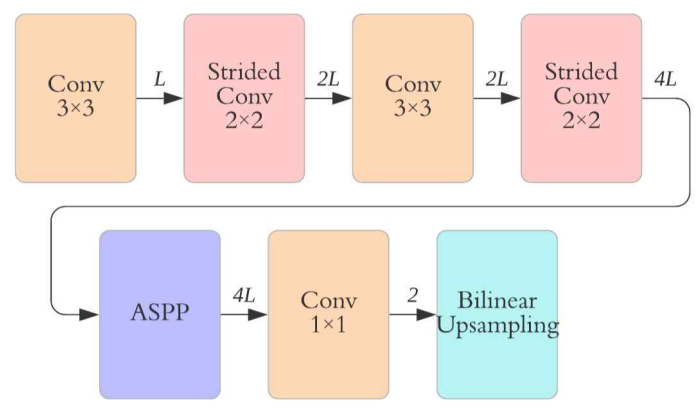

그림 3. DeepEye 모델 구조 [9] Fig. 3. DeepEye Model Architecture [9]
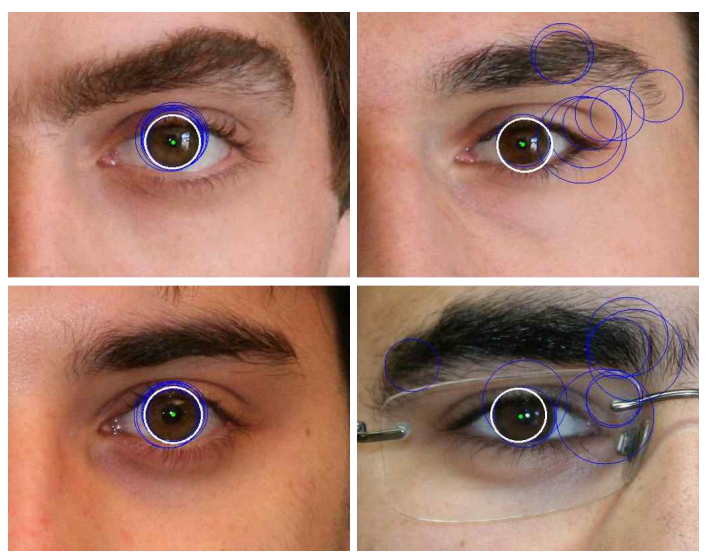

그림 4. 동공 중심점 기반 홍채 검출

Fig. 4. Iris Localization based on Pupil Center

DeepEye 모델을 통한 동공 중심 검출은 신경 망에서 곧바로 동공 중심 좌표를 추정하는 방식 은 아니다. DeepEye는 동공 주변의 각 픽셀이 동공 영역일 확률을 나타내는 마스크 영상을 추 정하고, 후처리를 통해 동공 중심 좌표를 계산하 는 방식을 사용한다. 그림 4 는 원형 허프 변환을 통해 검출된 홍채의 후보 영역과 함께, 검출된 동공 중심점을 기반으로 홍채의 검출 과정을 나 타낸 것이다. 그림에서 여러 개의 파란색 원은 홍채 후보 영역을 의미하며, 초록색 점은 검출된
동공 중심점을 의미하다. 하얀색 원은 동공 중심 점을 기준으로 후보 중에서 선택된 하나의 홍채 를 의미한다.

\subsection{AIPF 기반의 홍채 영역 보정}

원형 허프 변환(CHT)으로 검출된 홍채는 카메 라와 사람이 서로를 마주 보는 정면 영상일 경우 에는 비교적 정확하다. 그러나, 사람이 측면을 바 라보거나 카메라가 사람을 측면에서 촬영하는 경 우에는 영상에서 보이는 홍채의 모양이 원 모양 에서 점점 세로가 긴 타원 모양이 된다. 그리고, 카메라와 사람의 각도가 커질수록 검출 오차 또 한 커진다. 따라서 이러한 경우에 타원으로 홍채 를 검출하여야 더욱 정확하게 홍채 영역을 나타 낼 수 있다. AIPF(Angular Integral Projection Function)는 영상의 경계선을 검출하는 방법으로 기존의 $\mathrm{IPF}$ 에서 수평과 수직의 두 방향으로 탐 색하던 것을 모든 방향으로 확장한 것이다. 다음 식은 $\mathrm{AIPF}$ 의 경계선 검출을 위한 것이다[10].

$$
\begin{aligned}
& \operatorname{AIPF}(\theta, \rho, h)= \\
& \left|\begin{array}{l}
I\left(x_{0}+(\rho+h) \cos \theta, y_{0}+(\rho+h) \sin \theta\right) \\
-I\left(x_{0}+(\rho-h) \cos \theta, y_{0}+(\rho-h) \sin \theta\right)
\end{array}\right|
\end{aligned}
$$

식에서 $x_{0}, y_{0}$ 는 허프 변환에서 검출된 홍채의 중심 좌표를 의미하며, $\theta$ 는 중심 좌표를 기준으 로 탐색하는 각도를 의미한다. 또한, $\rho$ 는 현재의 탐색 반지름을 의미하는데, $\rho$ 의 탐색 범위는 1 부 터 $W$ 까지이다.

$\mathrm{AIPF}$ 의 탐색 과정은 그림 5 와 같다. 각 $\theta^{\text {에 }}$ 대하여 경계점을 추출하는데, 앞의 식은 $h$ 만큼 바깥에 있는 픽셀의 값과 안쪽에 있는 픽셀의 값 의 차를 계산한다. 이 값의 최대가 되는 픽셀이 경계점이라고 판단하며, 이때의 $\rho$ 값을 사용한다. 이와 같은 방식으로 모든 $\theta$ 에 대해 추출된 $\rho$ 값 을 통해 계산된 경계점은 타원 형태의 홍채를 검 
출하는 데 사용된다. 타원 형태의 홍채는 추출된 경계점에 대하여 최소자승법을 사용하여 검출되 는데, 최소자승법은 outlier에 민감한 특징을 갖 는다. 따라서, 추출된 경계점 중 outlier를 제거하 기 위해, 경계점의 중간값을 계산하고 중간값에 대해 모든 경계점의 차를 계산한 후에 최대 차와 의 비율이 0.4 보다 큰 것은 outlier로 판단하도록 하였다.

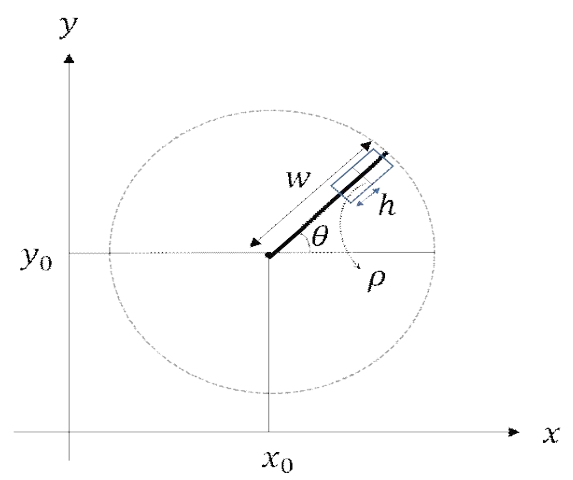

그림 5. AIPF 탐색 과정

Fig. 5. AIPF Exploration Process

\section{3. 실험 및 결과}

본 논문에서 제안한 홍채 검출 방법의 성능 정 확도를 확인하기 위하여, 공개 데이터셋을 사용 하여 실험을 진행하였다.

\subsection{UBIPr 데이터셋}

실험에 사용된 UBIPr 데이터셋[3]은 UBIRIS. v2 데이터셋[4]의 여러 버전 중 하나이며, 영상에 눈과 눈썹, 코 등의 주변부를 포함하고 있다. 또 한, 하나의 대상에 대하여 $4 \sim 8 \mathrm{~m}$ 사이의 다양 한 거리에서 촬영된 영상과 왼쪽, 정면, 오른쪽을 바라보는 영상이 포함되어 있다. 사용된 데이터 셋의 예제 영상을 그림 6에 나타내었다. 데이터
셋에 포함된 총 영상의 개수는 10,199 장이며, 실 험에서는 이 중에서 500 장의 영상을 임의 선택하 여 사용하였다.
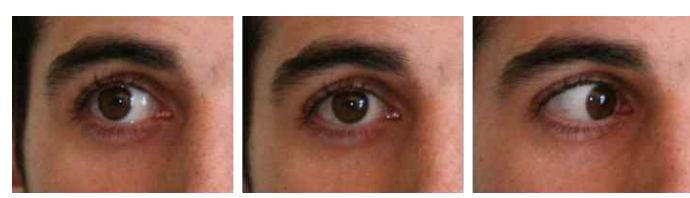

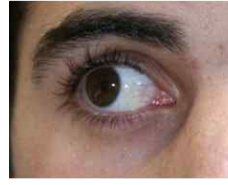

right

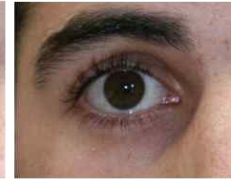

center

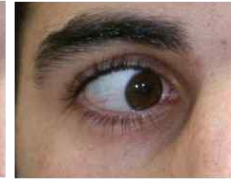

left
그림 6. UBIPr 데이터셋 예제

Fig 6. Examples of UBIPr Dataset

\section{2 실험 결과}

실험을 위하여, 기존 방법인 원형 허프 변환을 사용한 방법(CHT)과 제안된 DeepEye 기반의 동 공 중심 추정이 추가된 방법 $(\mathrm{HT}+$ DeepEye) 및 $\mathrm{AIPF}$ 기반의 보정을 추가한 방법 $(\mathrm{HT}+\mathrm{AIPF}$, $\mathrm{HT}+$ DeepEye + AIPF)에 대하여 성능을 측정 및 비교하였다. 성능 측정은 시각적 검사 방법을 사용하여, 검출된 홍채가 실제의 홍채와 일치하 는 경우에는 1 , 검출된 홍채가 실제 홍채와 완전 히 일치하지는 않지만, 동공을 포함하고 있는 경 우에는 0.5 , 앞의 두 가지 경우에 해당하지 않으 면 0 으로 계산하였다. 표 1은 이러한 방법으로 각각의 알고리즘에 대한 성능 측정 실험 결과이다.

표 1. 실험 결과

Table 1. Experimental Results

\begin{tabular}{|c|c|c|c|}
\hline 사용 알고리즘 & $\begin{array}{c}\text { 측정 } \\
\text { 점 }\end{array}$ & $\begin{array}{c}\text { 처리 시간 } \\
(\mathrm{sec})\end{array}$ & $\begin{array}{c}\text { 정확도 } \\
(\%)\end{array}$ \\
\hline $\mathrm{CHT}$ & 344.0 & 1.38 & 68.8 \\
\hline $\mathrm{CHT}+$ DeepEye & 410.5 & 1.41 & 82.1 \\
\hline $\begin{array}{c}\mathrm{CHT}+\mathrm{DeepEye}+ \\
\mathrm{AIPF}\end{array}$ & 481.0 & 2.05 & 96.2 \\
\hline
\end{tabular}


실험에 사용된 500 장의 데이터에 대하여 기존 방법인 $\mathrm{CHT}$ 의 정확도는 $68.8 \%$, 제안된 딥러닝 기반의 동공 검출 결과를 함께 사용한 방법 (CHT + DeepEye)은 $82.1 \%$ 로 측정되었다. 또한, 후처리를 추가한 경우 $(\mathrm{CHT}+$ DeepEye $+\mathrm{AIPF})$ 는 $96.2 \%$ 로 정확도가 향상됨을 확인하였다. 즉, $\mathrm{CHT}$ 와 동공 중심 검출 방법을 함께 사용하였을 때 약 $13.3 \%$ 의 성능 향상, 검출된 홍채를 타원으 로 보정하는 방법을 추가로 적용하였을 때 약 $14.1 \%$ 의 추가 향상된 결과를 보였다. 따라서, 동 공 중심점을 기반으로 홍채를 검출하고, 타원 형 태로 보정하는 제안된 방법이 원형 허프 변환 기 반의 방법에 비해 전체적으로 약 $27.4 \%$ 만큼 향 상된 정확도로 홍채를 검출할 수 있음을 확인하 였다. 그림 7 은 각각의 방법을 사용하여 검출된 홍채 영역을 나타낸 것이다. 검출된 홍채를 정확 히 비교하기 위해 원 영상에서 안구 영역만 확대 하여 제시하였다.

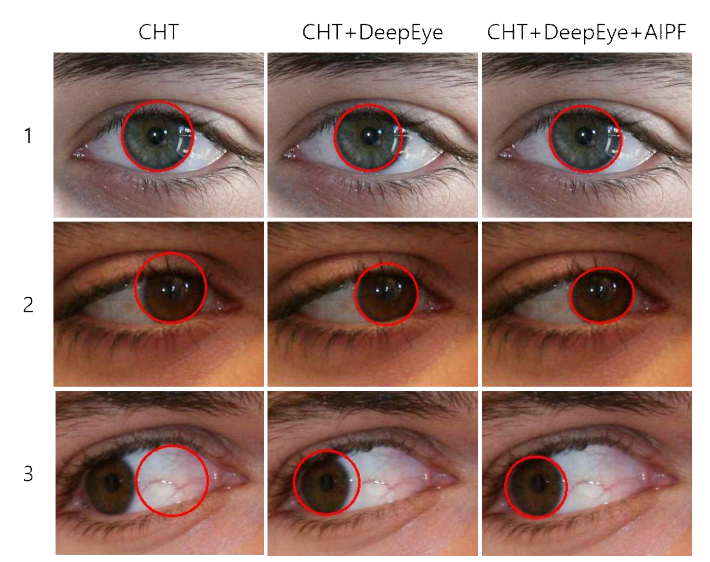

그림 7. 정확도 비교 예제

Fig. 7. Examples of Accuracy Comparison

한편, 검출 성능과 처리 시간과의 관계를 확인 하기 위하여, 각각의 방법에 대한 처리 시간을 측정하였다. 실험은 Intel Xeon(E5-2687W) CPU 2개와 NVIDIA GPU(GTX $1080 \mathrm{Ti})$ 2개가 설치
된 환경에서 수행되었으며, 실험에 사용된 데이 터셋 전체를 대상으로 처리 시간을 측정한 후 평 균값을 계산하였다. 표 1 에 제시된 것과 같이 $\mathrm{CHT}$ 의 처리 시간은 평균 1.38 초이며, DeepEye 기반의 동공 중심 검출이 추가되면 $30 \mathrm{~ms}$ 증가하 여 평균 1.41 초가 소요되었다. 그리고, $\mathrm{AIPF}$ 가 추가된 처리 시간은 $640 \mathrm{~ms}$ 증가한 평균 2.05 초이 다. 그러나 모든 알고리즘은 Python으로 구현되 었으며, DeepEye를 제외한 나머지 연산은 $\mathrm{GPU}$ 를 사용하지 않으므로 제시된 결과는 최적화를 통하여 추가적인 처리속도의 개선이 가능하다.

\section{4. 결 론}

본 논문에서는 원거리에서 촬영된 $\mathrm{RGB}$ 영상 에서 홍채를 검출하는 방법에 대하여 기술하였 다. 즉, 입력 영상의 에지 값에 대해 원형 허프 변환을 통해 홍채를 검출하는 기존의 방법에 딥 러닝 기반으로 검출된 동공 중심을 기준으로 여 러 홍채 후보 중 하나의 홍채를 선택하는 방법을 사용하였다. 또한, 후처리를 통해 원형으로 검출 된 홍채를 타원 형태로 보정하여 측면을 바라보 는 영상 등에서 다소 부정확한 검출 성능을 보완 하였다. 실험을 통해 제안된 방법이 기존 방법 대비 향상된 정확도로 홍채를 검출함을 확인하였 다. 제안된 방법을 활용하면 기존의 적외선 영상 기반 홍채인식 및 시선 검출을 $\mathrm{RGB}$ 영상에서도 적용할 수 있을 것이다.

이 논문은 2020년도 정부(과학기술정보통신 부)의 재원으로 수행된 연구임. (2019-000330, 영유아/아동의 발달장애 조기선별을 위 한 행동·반응 심리인지 $\mathrm{AI}$ 기술 개발) 


\section{참 고 문 헌}

[1] Kevin W. Bowyer and Mark J. Burge, Handbook of Iris Recognition, 2nd Eds. Springer, 2016.

[2] 고종국, 유장희, "원거리 홍채인식 기술 동 향”, 전자통신동향분석, 28권3호, pp.67-75, 2013.06.

[3] C. N. Padole, and H. Proenca, "Periocular Recognition: Analysis of Performance Degradation Factors", IAPR International Conference on Biometrics, pp.439-445, New Delhi, India, Mar. 2012. DOI: https://doi.org/10.1109/icb.2012.6199790

[4] H. Proenca, S. Filipe, R. Santos, J. Oliveira, and L. A. Alexandre, "The UBIRIS.v2: A Database of Visible Wavelength Iris Images Captured On-the-Move and At-A-Distance", IEEE Transactions on Pattern Analysis and Machine Intelligence, vol.32, no.8, pp.1529-1535, 2009. DOI: https://doi.org/ 10.1109/tpami.2009.66

[5] Z. Zhao and K. Ajay, "An Accurate Iris Segmentation Framework under Relaxed Imaging Constraints using Total Variation Model", in Proceeding of the IEEE International Conference on Computer Vision, pp.3828-3836, Boston, USA, Jun. 2015. DOI: https://doi.org/10.1109/iccv.2015. 436

[6] J. Daugman, "Statistical Richness of Visual Phase Information: Update on Recognizing Persons by Iris Patterns", Int'l Journal of Computer Vision, Vol. 45, No. 1, pp. 25-38, 2001.

[7] Li Ma, TieniuTan, YunhongWang, and
DexinZhang, "Efficient Iris Recognition by Characterizing Key Local Variations", IEEE Transactions on Image Processing, Vol 13, No. 6, pp. 739-750, Jun. 2004. DOI: https://doi.org/10.1109/tip.2004.827237

[8] M. Arsalan, H. G. Hong, R. A. Naqvi, M. B. Lee, M. C. Kim, D. S. Kim, and K. R. Park, "Deep Learning-based Iris Segmentation for Iris Recognition in Visible Light Environment", Symmetry, vol.9, no.11, pp.263, Nov. 4, 2017. DOI: https://doi.org/10.3390/sym9110263

[9] F. J. Vera-Olmos, E. Pardo, H. Melero, and N. Malpica, "DeepEye: Deep Convolutional Network for Pupil Detection in Real Environments", Integrated Computer-Aided Engineering, vol.26, no.1, pp.85-95, Dec. 3, 2018. DOI: https://doi.org/10.3233/ica-180584

[10] G. J. Mohammed, B. R. Hong, and A. A. Jarjes, "Accurate Pupil Features Extraction based on New Projection Function", Computing and Informatics, vol.29, no.4, pp.663-680, 2012.

[11] L. C. Chen, G. Papandreou, I. Kokkinos, K. Murphy, and A. L. Yuille, "Deeplab: Semantic Image Segmentation with Deep Convolutional Nets, Atrous Convolution, and Fully Connected CRFs", IEEE Transactions on Pattern Analysis and Machine Intelligence, vol.40, no.4, pp.834-848, 2018. DOI: https://doi.org/ 10.1109/tpami.2017.2699184

[12] N. Cherabit, F. Z. Chelali, and A. Djeradi, "Circular Hough Transform for Iris Localization", Science and Technology, vol.2, no.5, pp.114-121, 2012. DOI: https://doi.org/10.5923/j.scit.20120205.02 
저 자 소 개

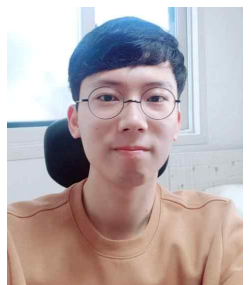

이태 균(Tae-Gyun Lee)

2018.02 한밭대학교 컴퓨터공학과 졸업 2020.08 과학기술연합대학원대학교

$\mathrm{ICT}$ 전공 석사과정 졸업

2020.09-현재 : 과학기술연합대학원대학교 $\mathrm{ICT}$ 전공 박사과정

<주관심분야> 인공지능, 딥러닝, 영상처리

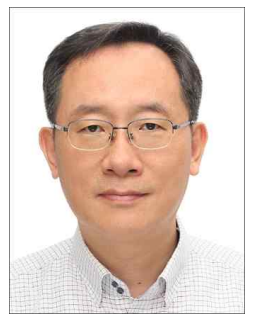

유장희(Jang-Hee Yoo)

1988.02 한국외국어대학교 물리학과 졸업 1990.02 한국외국어대학교 전산학과 석사

2004.07 영국 University of Southampton 전자 및 컴퓨터과학 박사

1989.11-현재: 한국전자통신연구원

인공지능연구소 책임연구원

2005.09 현재: 한국저작권위원회 감정인, 감정위원

2007.03-현재: 과학기술연합대학원 대학교

$\mathrm{ICT}$ 전공 전임교수

2007.01 현재: 한국SW감정평가학회 이사

2014.01 현재: 경찰청 과학수사자문위원

2014.8 2015.8: University of Washington 방문학자

2018.3 2020.3: 국가지식재산위원회 전문위원

<주관심분야> 컴퓨터 비전, 인공지능, 생 체인식, 휴먼모션분석, $\mathrm{HCI}$ 및 지능형 로봇 